\title{
Post-Traumatic Frontal Mucocele: Exceptional Cause and Frequent Recurrence
}

\author{
Hamid Khay, Mohammed Khoulali, Younes Dehneh, Lhamlili mohammed, Nabil Raouzi, Noureddine Oulali, Fayçal Moufid \\ Department of Neurosurgery, Mohammed VI university Hospital, Oujda, Morocco
}

\begin{abstract}
The frontal mucocele is a benign pathology characterized by its recurrence and is sometimes poor prognosis by the complications arising. We report the case of a young man, followed for recurrent frontal mucocel, secondary to a sinus trauma. It is managed surgically with multiple reccurence. Our goal is to decry the appearance intraoperative an exceptional case mucocel frontal posttraumatic recurrent and superinfected.
\end{abstract}

Keywords:- Post-Traumatic Mucocele, Surgery, Recurrence, Infection.

\section{INTRODUCTION}

Mucocele is a benign and rare cystic tumor, adults are mainly affected. The frontal and / or ethmoidal mucocele, despite its histological benignity, has an aggressive potential towards the local structures. It is due to a nonventilatilated sinus. The post-traumatic cause is exceptional and only one iatrogenic case reported following endonasal surgery. Surgical treatment consists of excision of the cyst and drainage of the causal sinus. ${ }^{1-3}$

\section{CASE REPORT}

A 38-years-old male patient with a history of nasal fracture three years before, the patient was operated ten months ago for mucocele revealed by left eye exophthalmos. The patient actually presents recurrence of his exophthalmos. Clinical examination revealed left eye exophthalmos non pulsatile, non-axial and irreducible with limitation in eye movement upward and inward. Nasal endoscopy showed nasal mucosa inflamed absence of the left lower horn, synechiae on both side with purulent secretions (figure1). MRI And CT-scan of the nasal sinus revealed a large left frontal mucocele lysing the superior orbital wall with orbito-palpebral extension hiding and driving the eyeball downwards and outside. The patient underwent surgery for drainage and marsupialization of the pathological frontal mucosa associated with excision of the frontal mucocele membrane, mucocele content were purulent, frontal sinus was aerated by milling the sinus walls. The patient also received intravenous antibiotic therapy based on amoxicillin. The postoperative marked by a total recovery of the visual function and almost normal eye-palpebral motility (Figure 2).

\section{CONCLUSION}

Surgical excision of mucocele and aeration the pathological sinus is the treatment of choice for mucocele and the recurrence one, mucocele is a benign tumor, but it can cause serious locoregional complications due the delay in treatment or recurrence problems, antibiotics only indicated in infected cases, as it was with our patient, crânio-facial trauma is common in developing countries including Morocco, close follow-up is indicated to detected these serious complications.

\section{REFERENCES}

[1]. Aghakhanyan G, Lupi G, Frijia F, et al. Delayed PostTraumatic Fronto-Ethmoidal Sinus Mucocele Evaluated with Short and Long TE MR Spectroscopy. Neuroradiol J. 26(6):693-698.

[2]. Gupta M, Chauhan B, Gupta M, Gupta AK. Large Frontoethmoidal Mucocele: Treatment and Review. doi:10.5005/JP-JOURNALS-10013-1171

[3]. Makihara S, Kariya S, Okano M, Naito T, Tsumura M, Nishizaki K. Orbital complications of infected mucocele in the paranasal sinuses. Auris Nasus Larynx. Published online June 11, 2020. doi:10.1016/j.anl.2020.05.012 


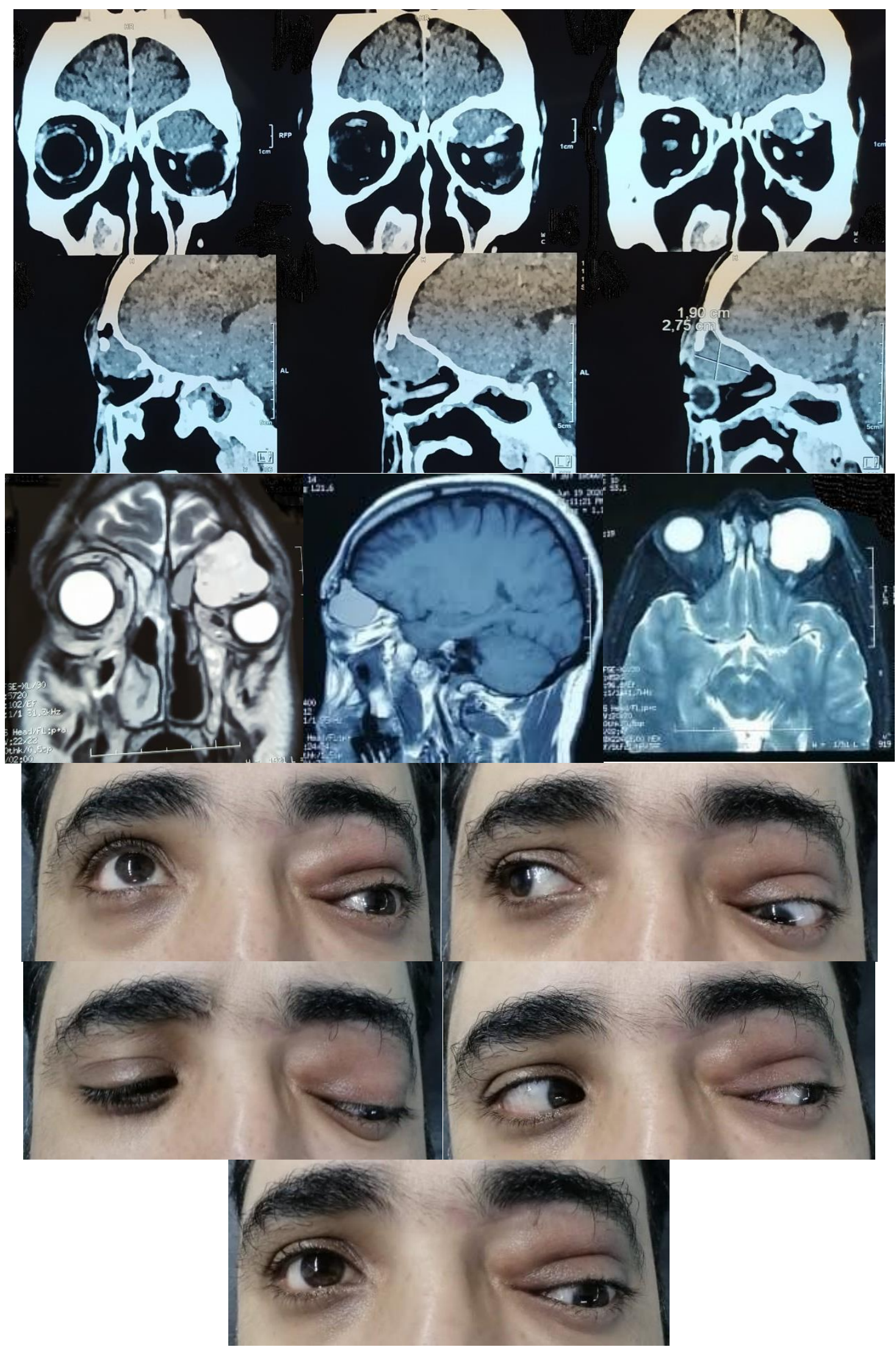

Fig 1:- preoperative images (permission obtained from the patient for publication) 
ISSN No:-2456-2165

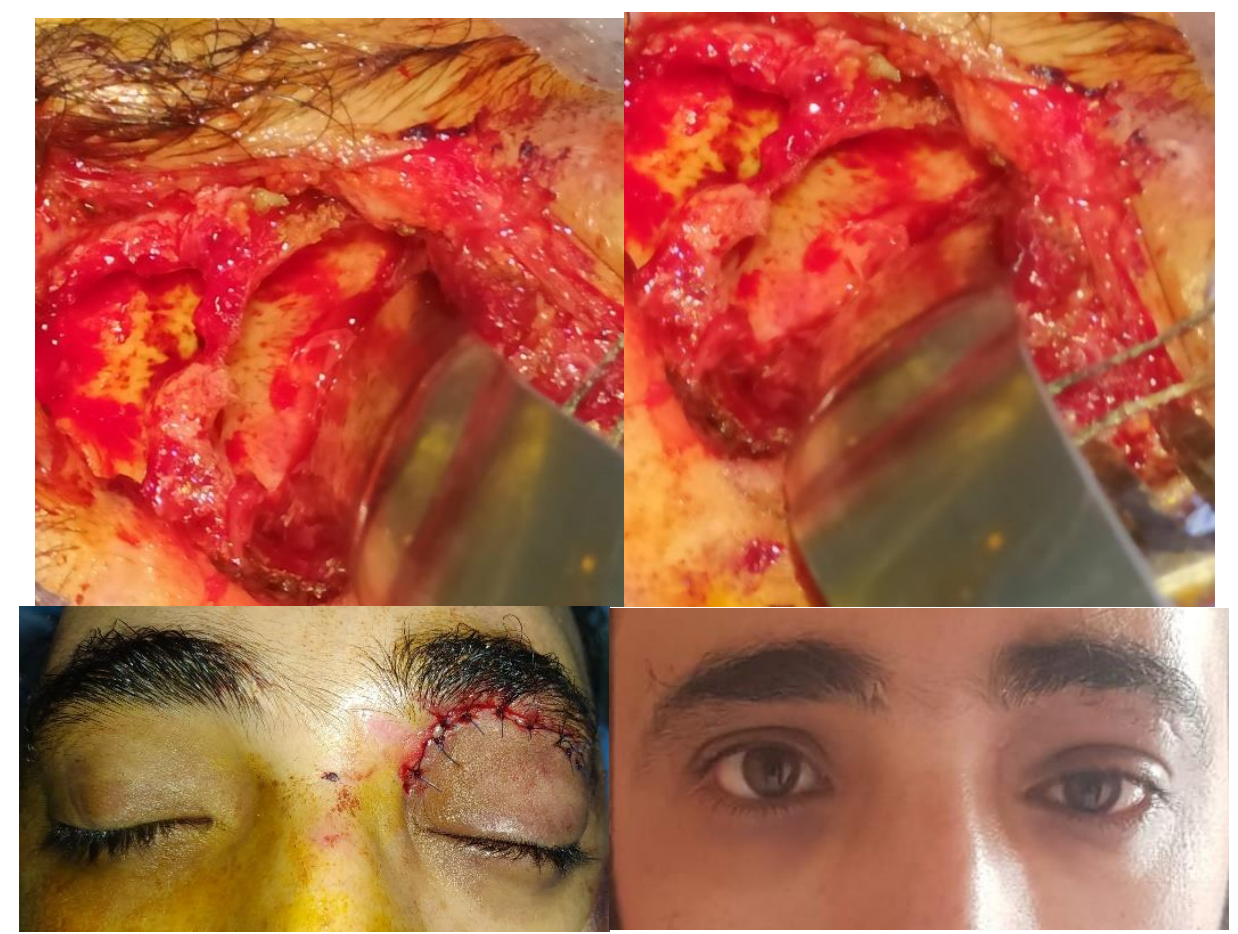

Fig 2:- operative and Postoperative images (permission obtained from the patient for publication)

Declarations:

- Ethics approval and consent to participate: Not applicable.

- Consent for publication: Not applicable.

- Availability of data and materials: Not applicable.

- Competing interests: The authors declare that they have no competing interests.

- Funding: None.

- Authors' contributions: Conception and design, Acquisition of data, Drafting the article, Reviewed submitted version of manuscript and Approved the final version of the manuscript: all authors.

- Acknowledgments: patient. 